\title{
Discrete element modelling of bendable tubes
}

\author{
Tom Leblicq*, Simon Vanmaercke, Herman Ramon, Wouter Saeys \\ KU Leuven - University of Leuven, Department of Biosystems (BIOSYST), Division of Mechatronics, Biostatistics and Sensors (MeBioS), Kasteelpark Arenberg \\ 30, 3001 Leuven, Belgium
}

\section{A R T I C L E I N F O}

\section{Article history:}

Received 21 November 2014

Received in revised form

21 January 2015

Accepted 24 February 2015

Available online

Keywords:

DEM

Simulation

Circular tube

Ovalisation

Buckling

\begin{abstract}
A B S T R A C T
Computer simulations are becoming increasingly important for the optimisation of various processes since they can significantly reduce the development time and costs. The discrete element method (DEM) offers a powerful tool for simulating the interactions of large numbers of particles. The method has proven its usefulness for many applications where granular and spherical particles are handled. Very recently DEM, was used to simulate the interactions of tubular particles. While the existing linear elastic models could describe the reversible bending and denting of these particles in a realistic way for small deformations, they are inadequate for describing the bending and compression behaviour at higher forces. In this region, buckling phenomena become important. Therefore, new phenomenological models, inspired by the collapse of steel tubes due to ovalisation of the cross section and the formation of plastic hinges, have been implemented for metal and synthetic plastic tubes. Using these models virtual, bendable tubes were created in the DEMeter ++ software. The DEM parameters were calibrated for copper and PVC tubes based on combined bending and denting tests conducted using an Universal Testing System. The simulations were in good agreement with the measurements $\left(R^{2}>0.91\right)$. This realistic DEM model of bending can, in future research, be used to simulate the processing of large numbers of tubular particles (e.g. the processing of crop stems).
\end{abstract}

(c) 2015 Elsevier Ltd. All rights reserved.

\section{Introduction}

Tubular particles are processed in various industries (e.g. the processing of biomass crops for energy production, the processing of fibers for the production of textiles, the processing of crops in agricultural machines, production of fibers for reinforcement of composites). For an optimal operation of these processes, quantitative insight in the interference of the particles, design and settings is required. Although empirical models can describe these processes well if they are calibrated with experiments, they cannot provide fundamental insight into the mechanical interactions of the particles and the influence of the boundary conditions. As this interaction takes place at the particle level, a modelling framework on this level should be set up.

\subsection{Discrete element modelling}

When modelling the behaviour of a collection of particles, discrete element modeling (DEM) is a logical choice as it allows us to model the behaviour of each particle through its interactions with the other particles and the system elements. In this way, the influence of particle properties and boundary conditions can be assessed with a set of in silico experiments that can be run in parallel [24].

\footnotetext{
* Corresponding author. Tel.: +3216 377116 .

E-mail address: tom.leblicq@biw.kuleuven.be (T. Leblicq).
}

DEM makes use of contact detection algorithms to determine which particles make contact. When particles interact, they are allowed to virtually overlap. The forces acting on these interacting particles are determined by a contact model and the virtual overlap. For each time step of the simulation, all forces acting on the particles are summed. Newton's equations of motion are then integrated to obtain the velocity and the position of each particle at the next time step [32].

DEM simulations can provide realistic insight in various processes if both the geometry of the particles and the contact models are sufficiently accurate. For rigid spherical particles (e.g. granular media) the capabilities of DEM have been shown in many studies $[5,1,27,26]$. However, few reports have been published on particles with large aspect-ratio, as simulating the movement and collisions of these particles is considerably more complex. Tubes in DEM should be compressible in both longitudinal and radial directions and also bendable in every direction to obtain realistic deformation behaviour.

\subsection{Tubular particles in DEM}

In the last years, tubular particles were created by a number of researchers $[31,7,10-13,21,8,15,29,28,24]$. The accuracy of the simulations with these particles varies greatly. Ross and Klingenberg [31] studied the dynamics of flowing suspensions of rigid and flexible fibres composed of linked rigid bodies. Favier et al. [7] developed a 


\begin{tabular}{|c|c|c|c|}
\hline \multicolumn{2}{|c|}{ Nomenclature } & C & curvature (-) \\
\hline$\delta$ & amount of flattening $(\mathrm{m})$ & $c_{h}$ & DEM damping coefficient for bending ( $\mathrm{Ns} / \mathrm{m}$ ) \\
\hline$\delta_{2}$ & $\begin{array}{l}\text { amount of flattening in the second phase of } \\
\text { buckling }(\mathrm{m})\end{array}$ & $\begin{array}{l}c_{t} \\
D\end{array}$ & $\begin{array}{l}\text { DEM damping coefficient for tension (Ns/m) } \\
\text { diameter }(\mathrm{m})\end{array}$ \\
\hline$\theta$ & bending angle (deg) & $D_{1}$ & diameter of the flattened tube $(\mathrm{m})$ \\
\hline$\theta_{b}$ & threshold angle for the start of buckling (deg) & $d$ & deformation of a spring damper-system (m) \\
\hline$\theta_{b 2}$ & $\begin{array}{l}\text { threshold angle for the start of the second phase of } \\
\text { buckling (deg) }\end{array}$ & $d_{\max }$ & $\begin{array}{l}\text { maximum deformation for all spring damper- } \\
\text { systems }(\mathrm{m})\end{array}$ \\
\hline$\nu$ & Poisson's coefficient $(-)$ & $k_{b}$ & DEM spring constant for bending $(\mathrm{N} / \mathrm{m})$ \\
\hline$\xi$ & dimensionless flattening $(-)$ & $k_{t}$ & DEM spring constant for tension $(\mathrm{N} / \mathrm{m})$ \\
\hline$\phi$ & mechanism angle for buckling (deg) & $r$ & radius $(\mathrm{m})$ \\
\hline$a_{2}$ & empirical parameter Eq. (13) $(-)$ & $t$ & wall thickness (m) \\
\hline$b$ & buckling parameter $(-)$ & $S$ & segment length $(\mathrm{m})$ \\
\hline$b_{2}$ & buckling parameter for the second & $X_{1}$ & see Fig. $3(\mathrm{~m})$ \\
\hline & buckling $(-)$ & $X_{2}$ & see Fig. $3(\mathrm{~m})$ \\
\hline
\end{tabular}

method for representing smooth-surfaced, axisymmetrical particles using overlapping spheres of arbitrary size. Virtual straw stems were developed by Kattenstroth et al. [21] by connecting spheres [7]. The stems in this research were, however, rigid and unbendable. The same method was used to create rigid fibres and rodlike particles $[10,11,28]$. Flexible filamentous particles composed out of chains of rigid bodies connected through ball and socket joints were developed by Geng et al. [8], Guo et al. [12,13], Grof and Štěpánek [11]. Guo et al. [15] compared glued-spheres particles and true cylindrical particles and concluded that for smooth particles the most realistic results are obtained with cylindrical particles. Guo et al. [14] also described methods for more optimal contact detection and dense packing of cylindrical particles. Lenaerts et al. [24] created segmented bendable straw stems. In this study the DEMeter ++ software was used [32]. The virtual stems were used for simulating grain-straw separation and the results were validated with measurements. Plant stems are basically tubes with small $D / t$-ratio's and low flexural stiffness. For this reason, the DEMeter ++ software was also used in this study.

In the DEM model of Lenaerts et al. [24] the virtual tubes consist of several segments (cylinders) which are connected by spherical joints. Since the cylinders are rigid, the joints are responsible for the flexibility of the tube. In every joint a number of spring-damper systems (Kelvin-Voigt models) is responsible for resisting the bending. Since the deformations were modelled as linear spring-damper systems, the tubes exhibit linear elastic behaviour when they are bent. This approximation is sufficient for small forces and deformations (as was the case in the experiments of Lenaerts et al. [24]). However, it has been shown that the bending behaviour of tubes is essentially non-linear $[3,4,25,6]$.

\subsection{Deformation of a tube during bending}

When a circular tube is bent, this results in longitudinal compression of the inner side of the tube and longitudinal tension in the outer side. Together, these forces determine the resistance to the applied bending moment. As both forces have a component in the direction of the center of the tube, the original circular cross section is flattened into an oval from. This initial elastic phase is therefore referred to as ovalisation. Ovalisation of circular tubes was first described by Brazier [3]. As the cross-section of the tube progressively becomes more oval the flexural stiffness of the tube reduces.

The way in which a tube reacts to this reduced bending resistance is dependent on the $D / t$-ratio. For large $D / t$-ratios short wavelength rippling will occur during bending (both for elastic [17] and inelastic cylinders [22]). At increasing bending angles the tube will locally collapse and a number of "diamond" shapes will be formed. For increasing wall thickness's (smaller $D /$ t-ratios) the ripples will occur at progressively higher curvatures and the collapse mode is characterised by one local sharp kink. For even thicker shells limit load instabilities are recorded first $[22,18]$.

When a tube with small $D / t$-ratio is bent, there is a point when the maximum bending force is passed and the structure becomes unstable. At this point a kink is formed and the cross-section locally completely flattens $[25,6,30]$. The tube then offers virtually no resistance to bending [4] and the tube buckles. So, buckling is not the result of an initial imperfection, but it is a result of the non-linear bending behaviour. Brazier [3] used an elastic energy approach to describe the dimensionless flattening of the tube and to determine the moment at which buckling occurs. He showed that this moment is independent of any parameter defining the tube. When reanalysing the problem Calladine [4] and Karam [19] showed that the true buckling moment is always lower than the Brazier moment. These researchers assumed that the cross-section deforms in-extensionally, which is mostly applicable for materials with a low Young's modulus [6]. For materials with larger elastic moduli Elchalakani et al. [6] divided the ovalisation phase in two parts: the first part is linear elastic bending of the tube without deformation of the cross-section. In the second part the bending moment stays constant and the crosssection ovalises to the point of buckling. The theoretical analysis of Brazier [3], Calladine [4] and Karam [19] stopped at this point, while Elchalakani et al. [6] showed that for steel tubes ovalisation does not stop when buckling starts, but continues throughout the entire bending process.

The collapse of a tube due to buckling involves the formation of a plastic hinge line. At this line the cross-section is flattened. This flattening also occurs, to a lesser degree, further away from the hinge line. Elchalakani et al. [6] improved the buckling model by Mamalis et al. [25] and presented a closed-form solution for describing the buckling behaviour of circular tubes. They assumed a linear relation between the flattening and the distance from the hinge line. In addition to concentrated plastic deformations at the hinge line tubes keep experiencing ovalisation over the length of the tube.

\subsection{Contributions of this study}

The discrete element method offers a powerful tool for modelling the interaction of large numbers of particles. The interaction of tubular particles with different dimensions and mechanical properties has already been simulated in DEM. For small forces and deformations the linear elastic method of Lenaerts et al. [24] provided good results. It has, however, been shown that bending of tubes is essentially nonlinear and plastic. So, to also obtain realistic simulations when the 
interactions between particles yield larger forces and deformations, this non-linear and plastic bending behaviour of the tubes should be incorporated. The aim of the presented study is to create virtual tubes which bend realistically due to interactions with other tubes and machine components.

First, it is discussed how linear elastic bendable tubes are modelled in DEMeter ++ . Then, phenomenological models are developed for describing ovalisation and buckling. Afterwards measurements are conducted on copper and PVC tubes for validation and for parameter estimation. Finally, a sensitivity analysis is conducted to gain insight in the influence of the different model parameters.

\section{Material and methods}

Each segment of a virtual tube in DEMeter ++ consists of a rigid hollow cylinder. Adjacent cylinders are connected by spherical joints. At both ends of the tube an additional joint is placed (Fig. 1). At each intersection between a cylinder and a joint a virtual disk is placed. Every joint consists thus of two disks. These two disks are interconnected with a number of linear, parallel spring-damper systems (Kelvin-Voigt models). The spring-damper systems are positioned in an axisymmetric pattern at a distance, equal to the radius of the tube, from the centre $((*)$ in Fig. 2). The stiffness of the springs $\left(k_{b}\right)$ and the distance of the spring to the centre of the tube determine the bending resistance of the joint. The dampers (with damping coefficient $c_{b}$ ) prevent the joint from oscillating, dissipate energy and stabilise the simulation. When bent, the spring-damper systems on the inner side are compressed. On the outer side they are stretched out.

In the research of Lenaerts et al. [24] the tubes consisted of five segments and six spring-damper systems per joint. The number of segments and spring-damper systems was, however, chosen arbitrary. While a larger number of segments per unit length results in a more realistic bending behaviour, the computational cost scales quadratically with this number. The optimal number of segments should, therefore, be determined for every application as a trade-off between accuracy and calculation time.

While the focus of this study is on the bending characteristics of tubes, other deformations may also play an important role during bending. Therefore, these deformations were also taken into account. The tensile stiffness of each segment is provided by an extra spring $\left(k_{t}\right)$ and damper $\left(c_{t}\right)$. They are attached to the two disks from one particular segment (Fig. 1). During three point bending the curvature is not only increased, the tube also experiences lateral forces at the plunger and the support points. The same phenomenon is observed when the bending of a tube is caused by the interactions with other tubes. Lateral forces can deform a cylinder in a process called denting. During this process the cross section is flattened $[35,20,2]$. To account for the lateral forces, per segment and per joint, an extra set of Kelvin-Voigt systems $\left(k_{r}\right.$ and $\left.c_{r}\right)$ is added in the radial direction [24]. Finally, friction forces can play a role. Due to the deflection the tube is

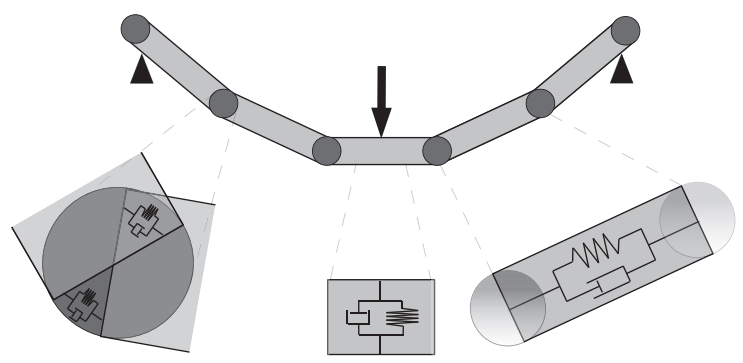

Fig. 1. A bendable tube in DEMeter ++ .

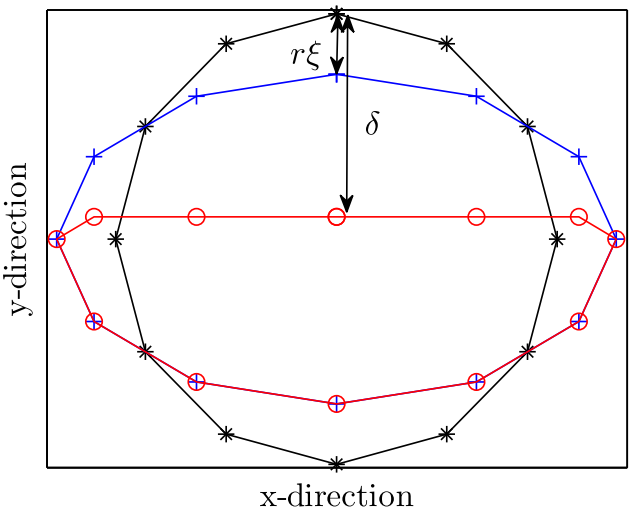

Fig. 2. Location of the Kelvin-Voigt elements $(N=12)$ on the virtual disk: $(*)$ undeformed cross-section, $(+)$ ovalised cross-section, (o) flattened cross-section.

shortened in the longitudinal direction. As a result, the tube will move on the supports. The Werner-Haff model [16] is used to model these tangential contact forces [32].

The mechanical models described in the literature for the bending and the denting of tubes often describe the observed phenomena and the force-deformation behaviour in a correct way. It is however not possible to directly plug these models in the DEM-software. The models can predict the bending behaviour of tubes as a whole, but in DEM the behaviour of individual elements and the interaction between these elements has to be known. For the presented DEM particles this means that the interaction of the different segments at the joints has to be determined. However, although the mechanical models cannot be implemented directly, they are very valuable as a basis for deriving phenomenological models to be included in DEM.

\subsection{Ovalisation}

Calladine [4] describes the ovalisation of an initial circular cross-section during bending. He found the following equation for the relation between the curvature $(C)$ and the dimensionless measure of the flattening in bending at the extreme fibre $(\xi)$ :

$\xi=\left(\frac{1-\nu}{t^{2}}\right) r^{4} C^{2}$

where $\nu$ is Poisson's coefficient, $t$ is the wall thickness of the tube and $r$ is the radius. During bending the curvature, and therefore also the ovalisation, increase. For this Eq. (1) to be useful it has to be adapted to the geometry, and the properties of the tubes in DEM.

The different segments of a tube in DEM are defined by a location and an orientation in the 3D space, by a segment length $(S)$ and by a diameter $(D)$. Different segments can interact in various ways (e.g. by joints between adjacent segments or by collisions between segments of different tubes). The DEMeter ++ software works in such a way that in each calculation step only the interaction between two segments is considered. When the tube is bent the two virtual disks intersect and some spring-damper systems are stretched while others are compressed. Since the location and the orientation of both segments are known, the angle $(\theta)$ between the segments and therefore also the amount of deformation $(d)$ of the spring-damper systems can be calculated (Fig. 3). By constraining the number of spring-damper systems to a multiple of four, there will always be two spring-damper systems where the deformation is largest. One spring-damper system will be compressed while the other one will be stretched out. This deformation is defined as $d_{\max }$. The relation between the maximum deformation $\left(d_{\max }\right)$ and the angle between 


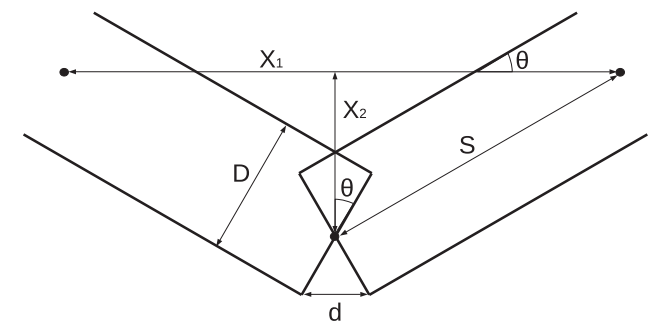

Fig. 3. Two segments of a bent tube in DEMeter ++ . (Each tube is defined by two points. Where the segments touch the points coincide.)

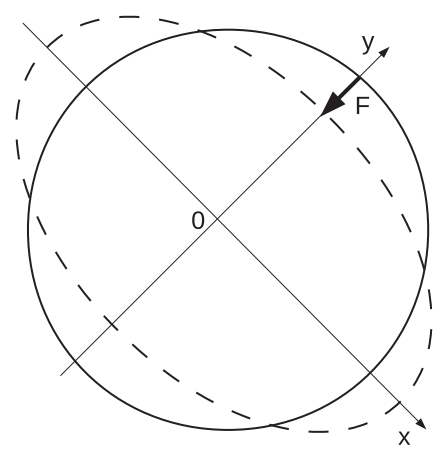

Fig. 4. Flattening of the initial circular cross-section (-, undeformed cross-section; - -, flattened cross-section).

the two segments is

$\sin (\theta)=\frac{d_{\max }}{D}$

The length $X_{1}$ of the line segment connecting the starting point of the first segment and the end point of the second segment decreased during bending, while the distance from that line segment to the intersection point between both segments $X_{2}$ increases

$X_{1}=2 S \cos (\theta), \quad X_{2}=S \sin (\theta)$

The Menger curvature for three non-collinear points is defined as the reciprocal of the radius of the circle that passes through the three points. It can be calculated using the following equation [23]:

$C(x, y, z)=\frac{1}{R}=\frac{4 A}{|x-y\|y-z\| x-z|}$

where $A$ denotes the area of the triangle spanned by $x, y$ and $z$. For the situation in Fig. 3 this gives

$C=\frac{\frac{4 X_{1} X_{2}}{2}}{S S X_{1}}=\frac{2 \sin (\theta)}{S}=\frac{2 d_{\max }}{S D}$

Substituting Eq. (5) in Eq. (1) gives

$\xi=p_{\text {ovalisation }} \frac{\left(\frac{D}{2}\right)^{2} d_{\max }^{2}}{S^{2}}$

where $p_{\text {ovalisation }}$ is a parameter defining the amount of the ovalisation, which is equal to $(1-\nu) / t^{2}$.

Before bending, the virtual tube is undeformed and all springdamper systems are axis-symmetrically arranged ((*) in Fig. 2). The cylinders defining the segments are rigid and can therefore not be deformed. The positions of the spring-damper systems in the joints are, however, not fixed. The configuration can be changed depending on the interaction of the segments. As mentioned before, this configuration will influence the bending resistance. For every time step and for every pair of segments the deformation of all springdamper systems is determined. The spring-damper system where the

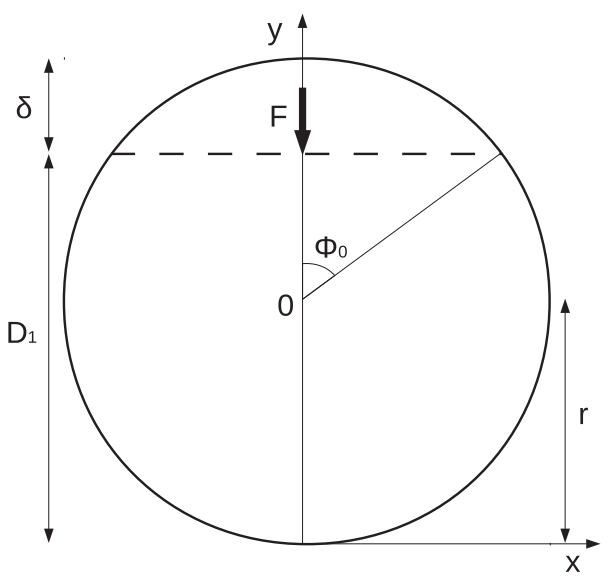

Fig. 5. Flattening of cross-section due to buckling (-; undeformed cross-section; - flattened cross-section).

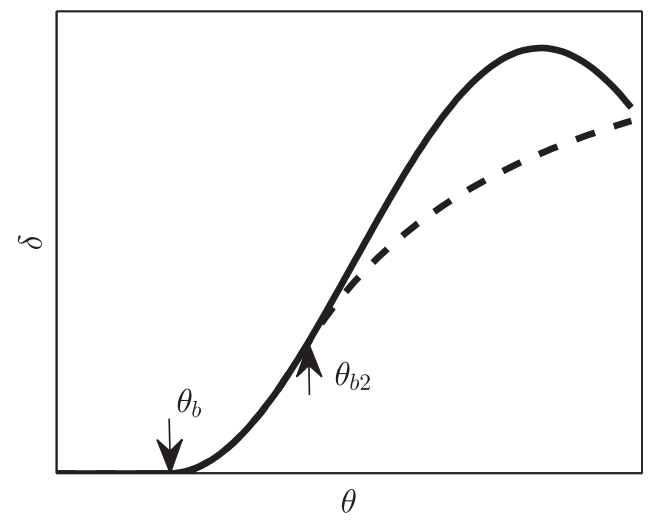

Fig. 6. Flattening of cross-section due to buckling ( - , model for the first phase of buckling Eq. (10); - -, model for the second phase of buckling Eq. (11)).

compression is the largest is also the spring-damper system closest to the external element causing the bending and the denting. From the value of this deformation $\left(d_{\max }\right)$ the dimensionless measure of the flattening $(\xi)$ can be obtained - see Eq. (6). Using this dimensionless flattening new positions can be calculated for the spring damper systems. The method for doing this is displayed in Fig. 4. A Cartesian coordinate system is applied to the initial configuration of the springdamper systems. The $y$-axis is determined by the origin and the spring-damper systems with the largest compression. The coordinate system is calibrated such that the $y$-coordinate of the spring-damper systems with the largest compression is equal to one. All springdamper systems are then moved in the direction of $y$ closer to the origin with an amount of $y_{n} \xi$ (were $y_{n}$ is the $y$-coordinate of the $n$th spring-damper systems). All spring-damper systems are also moved away from the origin in the perpendicular direction $(x)$ with an amount of $x_{n} \xi$ (were $x_{n}$ is the $x$-coordinate of the $n$th spring-damper systems). So, due to ovalisation the spring-damper systems are moved from position $\left(x_{n}, y_{n}\right)$ to $\left(x_{n}{ }^{\prime}, y_{n}{ }^{\prime}\right)$. In this way the total circumference stays constant. The result of ovalisation on the location of the spring-damper systems is displayed in Fig. $2(+)$.

Due to this ovalisation the bending resistance of the new configuration will be less than for the original one. It is clear that the amount of ovalisation and thus also the bending resistance is a function of both physical parameters $(t, D$ and $\nu$ ) and DEM parameters $\left(k_{b}\right.$ and $\left.S\right)$. Ovalisation and the reconfiguration of the spring-damper systems is defined elastic (and thus reversible) as was also assumed by Brazier [3] and Calladine [4]. This means that when the load, causing the bending, is removed all systems return to their original positions. 
Table 1

Dimensions of the measured tubes.

\begin{tabular}{lllllll}
\hline Tube parameters & Unit & \multicolumn{2}{c}{ Copper } & \multicolumn{3}{c}{ PVC } \\
\hline$D$ & $(\mathrm{~mm})$ & 8.24 & 10.16 & 15.05 & 16 & 20 \\
$t$ & $(\mathrm{~mm})$ & 0.64 & 0.76 & 0.86 & 1.49 & 1.62 \\
$D / t$ & $(-)$ & 12.9 & 13.4 & 17.4 & 10.7 & 12.3 \\
\hline
\end{tabular}

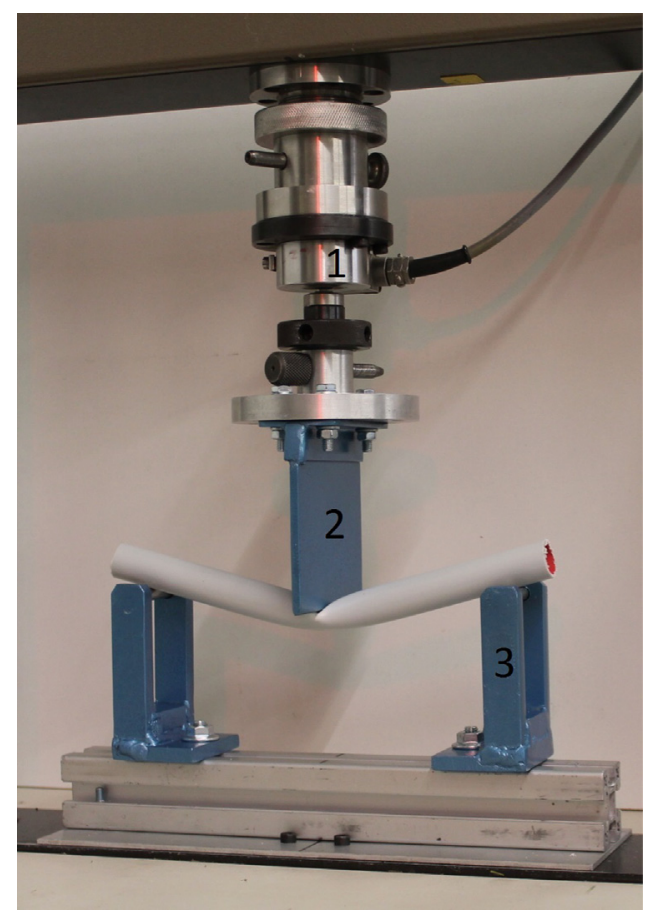

Fig. 7. Measurement set-up for combined bending and denting of PVC tubes consisting of (1) load cell and adopter, (2) plunger and (3) support.

\subsection{Buckling}

During ovalisation of the cross-section, the flexural stiffness of the tube decreases. After a certain threshold has been exceeded the structure becomes unstable and the tube locally flattens $[25,6,30]$. As previously stated this flattened cross-section offers less resistance to bending making the force deformation curve highly non-linear. The threshold can be expressed as the bending angle at which buckling takes place $\left(\theta_{b}\right)$. In Fig. 5 the result of the flattening in DEM is displayed schematically. Consistent with Elchalakani et al. [6], the angle $\phi_{0}$ is assumed to vary linearly with the applied bending angle $(\theta)$

$\phi_{0}=b \theta$

The parameter $b$ is a material parameter. In this study it is referred to as the buckling parameter. $D_{1}$ can be expressed as

$D_{1}=r\left(1+\cos \left(\phi_{0}\right)\right)$

The flattening $\delta$, due to buckling, can then be written as

$\delta=D-D_{1}=r\left(1-\cos \left(\phi_{0}\right)\right)$

Since buckling only occurs after a threshold angle $\left(\theta_{b}\right)$ has been exceeded:

$\delta=0 \quad$ if $\quad \theta<\theta_{b}$

$\delta=r\left(1-\cos \left(b \theta-b \theta_{b}\right)\right) \quad$ if $\quad \theta>\theta_{b}$

The cosine function in Eq. (10) makes this description of the flattening due to buckling periodic and would result in a decrease in the

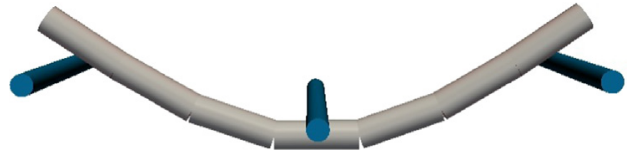

Fig. 8. Combined bending and denting test for tubes in DEMeter ++ .

deformation after reaching a maximum (Fig. 6). Such behaviour is, however, not realistic. Mamalis et al. [25], Elchalakani et al. [6] and Poonaya et al. [30] showed that flattening is plastic and increases with bending angle. Although the model is thus not suitable for large deformations it remains useful for smaller deformations. The transition between both phases takes place at the threshold angle $\left(\theta_{b 2}\right)$.

A second model is needed for the last phase of buckling. An empirical model, capable of describing the change in second moment of area of the cross-section in this phase, was sought

$\delta_{2}=a_{2} \frac{\theta}{\theta+b_{2}}+c_{2}$

$a_{2}, b_{2}$ and $c_{2}$ are empirical parameters. Since $\delta_{2}$ starts where $\delta$ stops and a continuous transition is imposed between the two models:

$\delta\left(\theta_{b 2}\right)=\delta_{2}\left(\theta_{b 2}\right)$

$d \delta\left(\theta_{b 2}\right)=d \delta_{2}\left(\theta_{b 2}\right)$

As a result of this constraint, the parameters $a_{2}$ and $c_{2}$ may be defined as

$a_{2}=\left[\frac{\left(\theta_{b 2}+b_{2}\right)^{2}}{b_{2}}-\theta_{b 2}\right] d \delta\left(\theta_{b 2}\right)$

$c_{2}=\delta\left(\theta_{b 2}\right)-a_{2} \frac{\theta_{b 2}}{\theta_{b 2}+b_{2}}$

Only one parameter $\left(b_{2}\right)$ remains. This parameter is the buckling parameter for the second phase of buckling. $\delta_{2}$ increases asymptotically to its maximum value of $\mathrm{D}$. It is, however, physically impossible to completely flatten the tube $(\delta=D)$ because of the wall thickness. For this reason $\delta_{2}$ is, in this research, limited to $D-2 t$. The tube retains thus at least the dimensions of the wall thicknesses. Physically, however, it is still possible to further compress the tube after this point. The effect of $\delta_{2}$ is displayed in Fig. 6 .

In this model the circumference of the tube is not kept constant which is in contrast to the assumptions made by Mamalis et al. [25], Elchalakani et al. [6] and Poonaya et al. [30]. While this is somewhat less accurate for small deformations, it will result in more realistic simulations. Additionally, the complexity of Eqs. (10) and (11) is much lower than that of the models described by Mamalis et al. [25], Elchalakani et al. [6] and Poonaya et al. [30]. This reduced complexity will be beneficial for the computing time.

The models for buckling and ovalisation both start from the configuration where all spring-damper systems are arranged axissymmetrically. The total deformation of the cross-section is the sum of the deformations by ovalisation and flattening by buckling. The effect of ovalisation is applied first, and only then the effect of buckling is taken into account. For buckling to have an effect on the position of a spring-damper system the effect of flattening in the $y$-direction must be larger than that of ovalisation. This is the case when $\delta>y_{n} \xi+\left(y_{n}-y_{n}^{\prime}\right)$. The new $y$-coordinate is then $y_{n}^{\prime \prime}=r-\delta$. If $\delta<y_{n} \xi+\left(y_{n}-y_{n}{ }^{\prime}\right)$ the $y$-coordinate is $y_{n}^{\prime \prime}=y_{n}{ }^{\prime}$. The effect of buckling on the configuration of the spring-damper systems is illustrated in Fig. 2 (o).

It is generally assumed that deformations due to ovalisation are elastic and that buckling is plastic $[3,4,6]$. As a consequence, the changes in the location of the spring-damper systems due to ovalisation are not permanent. When the force causing the deformation is removed, the systems return to their initial axis-symmetrical 
position. This is not the case for a reconfiguration of the springdamper systems due to buckling. These changes are permanent. Therefore, if the deformation is large enough to cause flattening by buckling the maximum deformation per joint is saved and used as the starting point for further deformation. The deformation can only increase when the configuration is further flattened.

When the proposed models for ovalisation and flattening are used only four parameters $\left(k_{b}, p_{\text {ovalisation, }} b\right.$ and $\left.b_{2}\right)$ and two
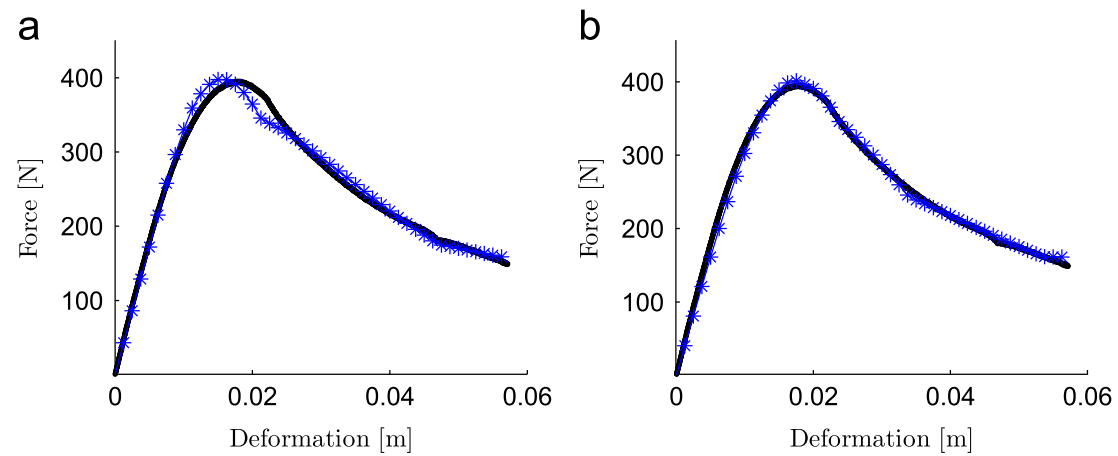

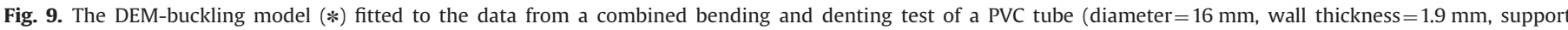
distance $=200 \mathrm{~mm}$ ): (a) five segments, 12 spring-damper systems, (b) seven segments, 20 spring-damper systems (blue: DEM simulation, black: measured data).

Table 2

Optimal DEM parameters (seven segments, 20 spring-damper systems).

\begin{tabular}{|c|c|c|c|c|c|c|c|c|c|c|c|}
\hline DEM parameters & Unit & Copper & & & & & & PVC & & & \\
\hline $\mathrm{L}$ & $(\mathrm{mm})$ & 200 & 200 & 200 & 300 & 300 & 300 & 200 & 200 & 300 & 300 \\
\hline$D / t$ & $(-)$ & 12.9 & 13.4 & 17.4 & 12.9 & 13.4 & 17.4 & 10.7 & 12.3 & 10.7 & 12.3 \\
\hline$k_{b}$ & $\left(10^{6} \mathrm{~N} / \mathrm{m}\right)$ & 0.945 & 1.34 & 1.592 & 0.666 & 1.20 & 1.34 & 0.242 & 0295 & 0.241 & 0.249 \\
\hline$p_{\text {ovalisation }}$ & $\left(1 / m^{2}\right)$ & 0.0029 & 0.0025 & 0.0024 & 0.0031 & 0.0022 & 0.0023 & 0.0032 & 0.0028 & 0.0031 & 0.0030 \\
\hline$\theta_{b}$ & (deg) & 1.21 & 1.22 & 1.22 & 2.64 & 1.53 & 1.23 & 0.55 & 0.49 & 0.65 & 0.852 \\
\hline$b$ & $(-)$ & 112.4 & 117.9 & 144.3 & 82.4 & 123.8 & 144.8 & 107.3 & 119.8 & 123.8 & 130.4 \\
\hline$\theta_{b 2}$ & (deg) & 2.13 & 1.97 & 2.05 & 3.31 & 2.87 & 2.73 & 1.06 & 0.98 & 0.97 & 1.02 \\
\hline$b_{2}$ & $(-)$ & 0.0176 & 0.0143 & 0.0130 & 0.0166 & 0.0151 & 0.0132 & 0.0320 & 0.0249 & 0.0339 & 0.0294 \\
\hline$R^{2}$ & & 0.93 & 0.99 & 0.91 & 0.94 & 0.97 & 0.94 & 0.99 & 0.93 & 0.97 & 0.91 \\
\hline
\end{tabular}
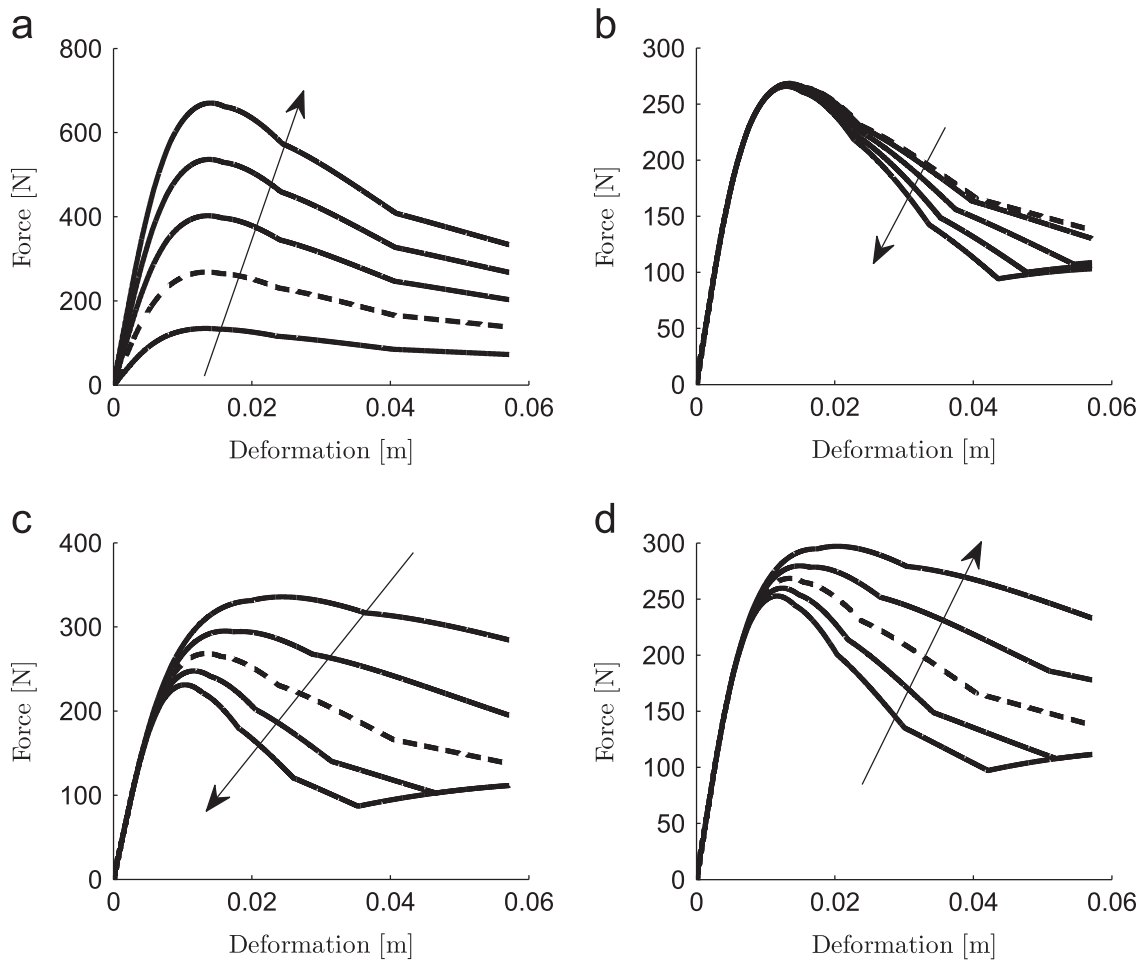

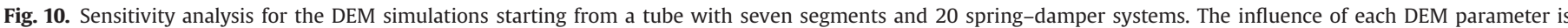

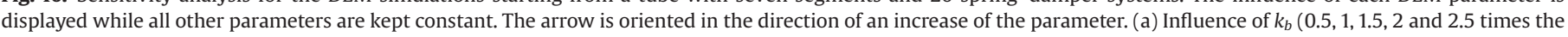

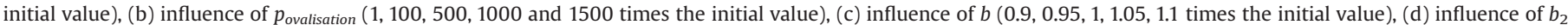
$\left(0.9,0.95,1,1.05,1.1\right.$ times the initial value). - -, initial value: $k_{b}=440,000, p_{\text {ovalisation }}=1000, b=100, b_{2}=0.05 ;-$, a multitude of the DEM parameter. 
thresholds $\left(\theta_{b}\right.$ and $\left.\theta_{b 2}\right)$ have to be specified to model the full bending behaviour of tubes in DEM. When the tubes are assumed to be homogeneous in their bending properties, only one set of parameters is needed to model all the segments and tubes in a simulation that can contain a large number of particles.

\subsection{Measurements}

To calibrate the DEM parameters and to validate the proposed methodology combined bending and denting tests were conducted on copper and PVC tubes of varying diameter and wall thickness (Table 1). For copper tubes three different commercially available $D / t$-configurations were tested. For PVC tubes two different $D / t$-configurations were tested.

The tubes were placed on two metal supports and then loaded midway with a metal plunger (Fig. 7). The set-up does not only bend the particles but also tends to indent them. This set-up was chosen because it corresponds to the way in which tubes are deformed in bulk DEM simulations, namely through the interaction with other tubes.

Each tube configuration was tested once at two support distances (200 and $300 \mathrm{~mm}$ ). For the copper tubes in total six configurations were tested. For the PVC tubes four configurations were tested. The tubes were cut as such that they were slightly longer than the support distance to avoid pushing off the tubes during bending. The plunger was driven by a universal testing system (UTS testsysteme $\mathrm{GmbH}$, type UTS $5 \mathrm{~K}$ ) at a loading rate of $1 \mathrm{~mm} / \mathrm{s}$. The location of the plunger was recorded during bending. The bending force was measured by a force transducer (Hottinger Baldwin Messtechnik $\mathrm{GmbH}$, type U2A $5 \mathrm{kN})$.

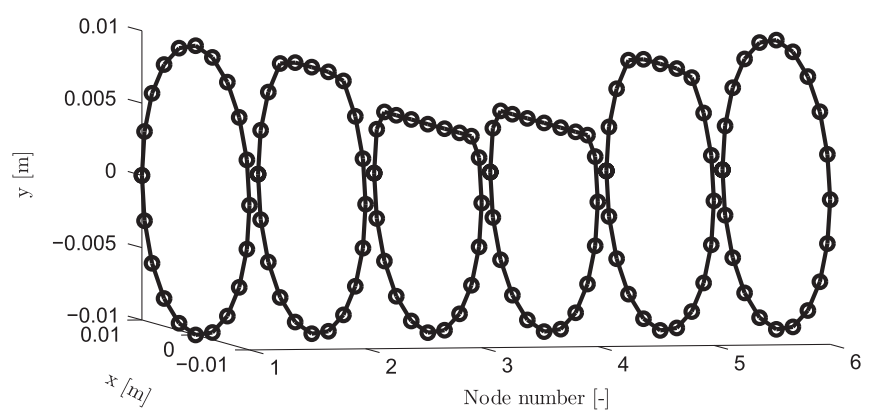

Fig. 11. Flattening of the initial circular cross-section.

\subsection{Estimation of DEM parameters}

For the calibration of the DEM parameters all 10 bending experiments were repeated with the virtual tubes. The diameter, the length and the mass per meter of the tubes, the support distance, the radius of the supporting points, the radius and the displacement of the plunger and the deformation rate were all giving the same values in DEM as in the physical experiments. In this study the number of segments and spring-damper systems per joint was chosen in such a way that the $R^{2}$-value for each configuration was higher than 0.9 . Tubes consisting of seven segments and 20 spring-damper systems per joint yielded this result (Fig. 8). For a increasing number of segments and spring-damper systems the simulation will convergence towards the physical solution. A larger number of segments and spring-damper systems will, however, result in a longer computation time. A time-step $(\Delta t)$ of $1 \times 10^{-6} \mathrm{~s}$ was used as this proved to be sufficiently small in order to obtain stable simulations. The force and the position of the plunger were determined for every time step. The force-deformation profiles from the physical experiments were compared to those of the computer experiments. The DEM parameters $\left(k_{b}, p_{\text {ovalisation }}, \theta_{b}, b, \theta_{b 2}, b_{2}\right)$ were then estimated iteratively. This optimisation was done using matlab (MATLAB and Optimisation Toolbox Release 2012b, The MathWorks, Inc., Natick, Massachusetts, United States).

Young's modulus for PVC is $3.38 \mathrm{GPa}$ [34], for copper it is $120 \mathrm{GPa}$ [9]. These material properties were used to derive the spring constants $k=F / \Delta L$ for the tensile $\left(k_{t}\right)$ and radial stiffness $\left(k_{r}\right)$ of the tubes [36]

$k_{t}=\frac{F}{\Delta L}=\frac{E \pi\left(r^{2}-(r-t)^{2}\right)}{L}$

$\Delta r=\frac{\left(\frac{\pi}{4}-\frac{2}{\pi}\right) \frac{12 F}{L E}}{\left(\frac{t}{r}\right)^{3}}$

$k_{r}=\frac{F}{\Delta r}=\frac{L E\left(\frac{t}{r}\right)^{3}}{12\left(\frac{\pi}{4}-\frac{2}{\pi}\right)}$

As each segment consists of a spring for tensile stiffness and one for radial stiffness, while the joints contain an extra spring for the radial stiffness, the total stiffness of the tube is the combined effect of these different springs. The spring constant for each individual spring in the radial direction should then be the total radial spring constant divided a

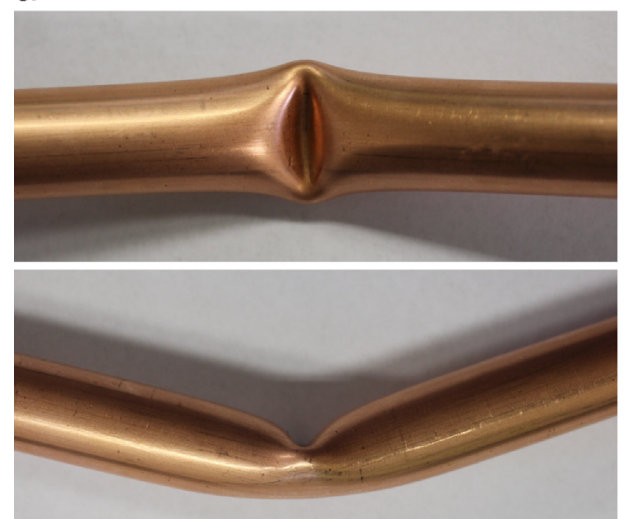

b

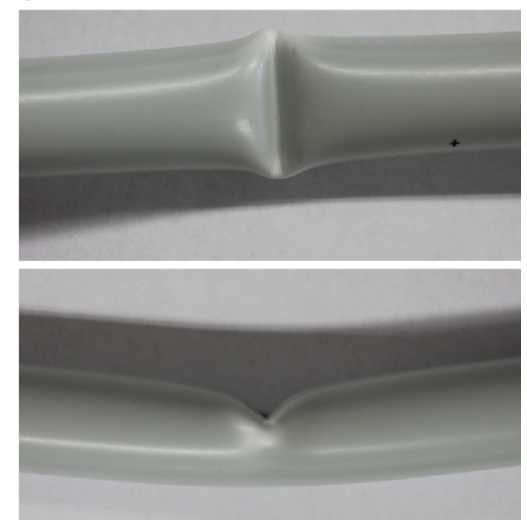

Fig. 12. Flattening of a (a) copper and (b) PVC tube due to bending. 
by the number of radial springs. The same is true for the tensile springs. For both the PVC and the copper tubes the friction coefficient $\mu$ with the steel of the support was set to 0.4 [9].

\section{Results and discussion}

In Fig. 9(b) the DEM force-deformation profile for a tube with seven segments and 20 spring-damper systems, corresponding best to the measured data from a combined bending and denting test of a PVC tube, is displayed. To demonstrate that the result is dependent on the number of segments and spring-damper systems, a configuration with five segments and 12 spring-dampers is also displayed (Fig. 9(a)). When the results obtained with both configurations are compared, it becomes clear that more accurate results are obtained when the number of segments and the number of spring-damper systems is increased. The good agreement between the measured and simulated curves show that the model is capable of describing the strong decrease in bending resistance due to flattening of the cross section. Good results were obtained for all measurements. A summary of the DEM parameters and the $R^{2}$-values is presented in Table 2 .

The spring-constant for bending $k_{b}$ increases with an increasing $D / t$-ratio for both copper and PVC tubes. Tubes with a larger $D / t$-ratio not only have a larger diameter but also a larger wall thickness (Table 1). This leads to an steeper initial force-deformation curve and a larger maximum force. In Table 1 it can also be seen that when the distance between the support points is increased the spring-constant decreases. A similar effect has been described for Young's modulus in bending [33]. The ovalisation parameter $\left(p_{\text {ovalisation }}=(1-\nu) / t^{2}\right)$ decreases with an increase in wall thickness. As expected the support distance has no influence on this parameter. No clear relation could be found between the threshold angles for buckling $\left(\theta_{b}\right.$ and $\left.\theta_{b 2}\right)$ and the $D / t$-ratio or the support distance. The buckling parameter $b$ increases with an increase of $D / t$, so for the same bending angle the tubes with a larger $D / t$-ratio will have a larger deformation $\left(\delta\right.$ ). The inverse relation between $\delta_{2}$ and $b_{2}$ could explain the decrease of $b_{2}$ with an increase of the $D / t$-ratio. No clear correlation could be observed between the support distance and the parameter values of $b$ and $b_{2}$.

\subsection{Sensitivity analysis}

The DEM model parameters are highly correlated. As a result, it is not possible to determine unaliased relations between the parameters and the force-deformation curve. To gain insight into the effect of the different parameters on the buckling process, a sensitivity analysis was conducted (Fig. 10). As a starting point the optimal DEM parameters for a copper tube with a diameter of $8 \mathrm{~mm}$ and a wall thickness of $0.6 \mathrm{~mm}$ were used. Each parameter was then varied while the remaining parameters were kept constant. As can be seen in Fig. 10 all parameters have an influence on the bending process. When the spring constant $\left(k_{b}\right)$ is increased both the initial stiffness, the maximum force and the force reduction rate due to buckling increase. For the tubes in this study the ovalisation parameter was very low, indicating that ovalisation is not so important. In a more elastic material (e.g. PET), the ratio can be different. Both buckling parameters $b$ and $b_{2}$ influence the force reduction rate. A larger value for $b$ results in more flattening of the cross section and thus a larger force reduction. The opposite is true for $b_{2}$. This follows directly from the inverse relationship between $b_{2}$ and $\delta_{2}$ - see Eq. (11)

The influence of ovalisation and buckling differs from joint to joint as the degree of flattening is determined for each joint, depending on the angle between the two adjacent segments and the DEM parameters. In Fig. 11 the effect of ovalisation and buckling on the location of the Kelvin-Voigt elements on the virtual disks is displayed for the six joints that connect two adjacent segments in a seven segment tube. The effect is largest in the central joints while the other joints are flattened to a lower degree. This is in agreement with the observation of reduced flattening further away from the hinge line [6]. This effect was also visually observed for the PVC and copper tubes in this study (Fig. 12). The fact that this effect was not intentionally inserted in the model, but follows automatically from the DEM simulations, confirms the potential of this model to realistically simulate the bending behaviour of tubes.

\section{Conclusions}

Phenomenological models, based on the existing mechanical knowledge, were developed to create virtual tubes with behave realistic when bent by the interaction with other tubes. The models take ovalisation and buckling phenomena, occurring during bending of tubes, into account. Combined bending and denting tests were performed on PVC and copper tubes to calibrate the DEM parameters and to validate the method. Tubes with different dimensions were tested at different support distances. A good agreement was found between measurements and simulations for all measurements $\left(R^{2}>0.91\right)$. A sensitivity analysis was conducted to gain insight in the effect of the different DEM parameters on the buckling process. The bending stiffness was found to influence the entire deformation profile. For copper and PVC tubes the ovalisation was found to play a minor role as these materials behave more plastic than elastic. Both buckling parameters influenced the force reduction rate due to buckling.

In the future this DEM model will be used to simulate processes where realistic bendable tubes are required. An example is the compression of biomass for ease of handling and transport. The bending of the plant stems is an integral part of the processing. It is now possible to investigate, in a realistic way, how different tubes interact with each other and with machine components. The simulations can be used to make a targeted choice on future improvements and to reduce test expenditures. It has become possible to estimate performance increases.

\section{Acknowledgements}

The authors wish to thank Dr. Bart Lenaerts for his assistance regarding the simulations and Dirk Leroy for constructing the test set-up. Also thanks to IWT/KU Leuven IOF for support in the development of Demeter ++ .

\section{References}

[1] van Baars S. Discrete element analysis of granular materials. Int J Rock Mech Min Sci Geomech 1996:41:139-57.

[2] Błachut J, Iflefel I. Experimental and numerical investigation of plain and gouged dents in steel pipes subjected to pressure and moment loading. J Press Vessel Technol 2008;130:021203.

[3] Brazier LG. On the flexure of thin cylindrical shells and other "thin" sections. Proc R Soc Lond 1927:104-16.

[4] Calladine CR. Theory of shell structures. New York, USA: Cambridge University Press; 1989.

[5] Cundall PA, Strack OD. A discrete numerical model for granular assemblies. Geotechnique 1979;29:47-65.

[6] Elchalakani M, Zhao XL, Grzebieta RH. Plastic mechanism analysis of circular tubes under pure bending. Int J Mech Sci 2002;44:1117-43.

[7] Favier J, Abbaspour-Fard M, Kremmer M, Raji A. Shape representation of axisymmetrical, non-spherical particles in discrete element simulation using multi-element model particles. Eng Comput 1999;16:467-80.

[8] Geng F, Li Y, Wang X, Yuan Z, Yan Y, Luo D. Simulation of dynamic processes on flexible filamentous particles in the transverse section of a rotary dryer and its comparison with video-imaging experiments. Powder Technol 2011;207:175-82.

[9] Gere JM. Mechanics of materials. Belmont, CA, USA: Thomson Learning, Inc.; 2004.

[10] Grof Z, Kohout M, Štěpánek F. Multi-scale simulation of needle-shaped particle breakage under uniaxial compaction. Chem Eng Sci 2007;62:1418-29. 
[11] Grof Z, Štěpánek F. Distribution of breakage events in random packings of rodlike particles. Phys Rev E 2013;88:1-5.

[12] Guo Y, Curtis J, Wassgren C, Ketterhagen W, Hancock B. Granular shear flows of flexible rod-like particles. POWDERS AND GRAINS 2013: In: Proceedings of the seventh international conference on micromechanics of granular media, vol. 1542; 2013. p. 461-94.

[13] Guo Y, Wassgren C, Hancock B, Ketterhagen W, Curtis J. Validation and time step determination of discrete element modeling of flexible fibers. Powder Technol 2013;249:386-95.

[14] Guo Y, Wassgren C, Ketterhagen W, Hancock B, Curtis J. Some computationa considerations associated with discrete element modeling of cylindrical particles. Powder Technol 2012;228:193-8.

[15] Guo Y, Wassgren C, Ketterhagen W, Hancock B, James B, Curtis J. A numerical study of granular shear flows of rod-like particles using the discrete element method. J Fluid Mech 2012;713:1-26.

[16] Haff P, Werner B. Computer simulation of the mechanical sorting of grains. Powder Technol 1986;48:239-45.

[17] Houliara S, Karamanos SA. Stability of long transversely-isotropic elastic cylindrical shells under bending. Int J Solids Struct 2010;47:10-24.

[18] Ju G, Kyriakides S. Bifurcation and localization instabilities in cylindrical shells under bending-ii. Predictions. Int J Solids Struct 1992;29:1143-71.

[19] Karam GN. On the ovalisation in bending of nylon and plastic tubes. Int J Press Vessels Pip 1994;58:147-9.

[20] Karamanos SA, Andreadakis KP. Denting of internally pressurized tubes under lateral loads. Int J Mech Sci 2006;48:1080-94.

[21] Kattenstroth R, Harms H, Lang H. Systematic alignment of straw to optimise the cutting process in a combine's straw chopper. In Proceedings of LandTechnik AgEng; 2011.

[22] Kyriakides S, GT J. Bifurcation and localization instabilities in cylindrical shells under bending-i. Experiments. Int J Solids Struct 1992;29:1117-42.
[23] Léger J-C. Menger curvature and rectifiability. Ann Math 1999;149:831-69.

[24] Lenaerts B, Aertsen T, Tijskens E, De Ketelaere B, Ramon H, De Baerdemaeker J, et al. Simulation of grain-straw separation by discrete element modeling with bendable straw particles. Comput Electron Agric 2014;101:24-33.

[25] Mamalis A, Manolakos D, Baldoukas A, Viegelahn G. Deformation characteristics of crashworthy thin-walled steel tubes subjected to bending. Proc Inst Mech Eng Part C: J Mech Eng Sci 1989;203:411-7.

[26] Martin C, Bouvard D, Shima S. Study of particle rearrangement during powder compaction by the discrete element method. J Mech Phys Solids 2003:51:667-93.

[27] Matuttis H, Luding S, Herrmann H. Discrete element simulations of dense packings and heaps made of spherical and non-spherical particles. Powder Technol 2000;109:278-92.

[28] Nan W, Wang Y, Ge Y, Wang J. Effect of shape parameters of fiber on the packing structure. Powder Technol 2014;261:210-8.

[29] Nguyen DH, Kang N, Park J. Validation of partially flexible rod model based on discrete element method using beam deflection and vibration. Powder Technol 2013;237:147-52.

[30] Poonaya S, Teeboonma U, Thinvongpituk C. Plastic collapse analysis of thin-walled circular tubes subjected to bending. Thin-walled Struct 2009;47:637-45.

[31] Ross RF, Klingenberg DJ. Dynamic simulation of flexible fibers composed of linked rigid bodies. J Chem Phys 1997;106:29-49.

[32] Tijskens E, Ramon H, De Baerdemaeker J. Discrete element modelling for process simulation in agriculture. J Sound Vib 2003;266:493-514.

[33] Timoshenko S, Gere JM. Theory of elastic stability. New York, USA: McGrawHill Book Company, Inc.; 1961.

[34] Titow WV. PVC technology. Essex, England: Springer; 1984.

[35] Wierzbicki T, Suh M. Indentation of tubes under combined loading. Int J Mech Sci 1988;30:229-48.

[36] Yamada R, Suzuki M, Harayama Y. Application of finite element method to ring compression test. Nuclear Eng Des 1977;44:75-85. 